\title{
PRESSURE LAWS AND FAST LEGENDRE TRANSFORM
}

\author{
P. HELLUY, H. MATHIS
}

\begin{abstract}
In this paper we investigate algorithms based on the Fast Legendre Transform (FLT) in order to compute tabulated Equation Of State (EOS) for fluids with phase transition. The equation of state of a binary mixture is given by an energy minimization principle. According to the miscible or immiscible nature of the mixture, the energy of the system is either a convex envelope or an inf-convolution of the energies of the two phases. Because these operations are closely linked to Legendre Transform, it is possible to construct $O(N)$ algorithms that compute efficiently these operations, where $N$ is the number of points in the table. In addition, it appears that the natural mathematical tool for studying mixture thermodynamics in the Legendre space is the max-plus algebra theory.
\end{abstract}

\section{INTRODUCTION}

We are interested in the modeling of compressible fluids with phase transitions. The evolution of such fluids is described by the Euler equations of gas dynamics. This system contains an Equation Of State (EOS) that relates the pressure of the fluid to the density and the energy. Thermodynamics require that the EOS satisfies some convexity constraints, which ensure, for instance, the hyperbolicity of the Euler equations.

The standard van der Waals equation is a classical EOS for fluids with phase transition. It is a well-known fact that this EOS is not globally convex. It causes the fluid equations to be of a mixed hyperbolic/elliptic type [MP89]. An admissible pressure law is obtained by applying the Maxwell equal-area rule. It appears that this construction is equivalent to compute the convex hull of the van der Waals energy. The same problem may appear when we wish to mix several phases with different EOS. Standard approximation tools, such as polynomial interpolations, generally do not preserve convexity and may lead to unphysical behavior.

The main result of our work is that the good mathematical framework for constructing admissible EOS is the theory of the so-called max-plus algebra [Ma87], which is itself closely related to the Legendre-Fenchel transformation. This tool is classically applied to optimal control problems or to probability theory. Once this framework is set, natural numerical algorithms can be constructed in order to properly approximate the EOS of real fluids.

The outlines of this paper are the following.

In the section 1, we recall the thermodynamics theory of a single fluid. The internal energy is a convex function of the entropy and the volume of the fluid, either in term of extensive or intensive parameters. Then, we investigate the thermodynamics theory of mixtures. First, we consider an immiscible mixture of two phases of a same pure body as in [HS06]. 
According to the minimum energy principle, the mixture energy turns out to be the inf-convolution of both energies, in extensive variables. When we we switch to intensive variables, the mixture energy is the convex hull of the minimum of the two energies. This property is studied by Faccanoni in her thesis [AFK07]. A similar analysis is performed here in the case of miscible mixtures. The mixture energy, in extensive or intensive variables, is obtained from an inf-convolution operation.

As it is noticed in [HS06], the equilibrium energy can be related to classical operations in convex analysis that are the inf-convolution and the Legendre transform. These two operations are closely linked: the inf-convolution is transformed into an addition by the Legendre transform. Moreover applying twice a Legendre transform to any function is an elegant way to compute the convex hull of a function. This operation has thus an interest in the computation of equilibrium state laws. In addition, like the Fourier transform, the Legendre transform can be computed by a fast algorithm with linear complexity (see [Br89], [Lu96], [Lu98]). In Section 2 general properties about the Legendre transform are recalled. We also detail a Fast algorithm to compute the Legendre Transform.

Section 3 is devoted to the numerical application of the FLT in the thermodynamical framework. First, we point out that the FLT can be used to perform in an easy way usual changes of variables. This trick is applied to the computation of isothermal curves. Then we compute the equilibrium of two phases satisfying the perfect gas law. The results are in agreement with those available in [Ja01] or [HS06]. Our algorithm is also applied to the van der Waals EOS: applying twice the Legendre transform is equivalent to a convexification process. It is also equivalent to apply the equal-area rule in the Maxwell construction. Finally, we propose a miscible mixture computation in order to illustrate the generality of our approach.

\section{Phase transition thermodynamics}

1.1. Single fluid thermodynamics. We consider a pure body of mass $M \geq 0$, entropy $S \geq 0$, occupying a volume $V \geq 0$. We assume the system to be entirely described by its state vector:

$$
W=(M, S, V) \in C .
$$

The set $C$ is the set of admissible states. It is a closed convex cone of $\mathbb{R}^{3}$ defined by

$$
C=\{W=(M, S, V), M \geqslant 0, S \geqslant 0, V \geqslant 0\} .
$$

Remark 1. The positivity of the entropy $S$ is ensured by the less known Nernst third law of thermodynamics (see [Ca85]).

It is classical in thermodynamics to distinguish between extensive and intensive quantities.

Definition 1. Let $F$ be an application from $C$ into $\mathbb{R}^{+}$. The quantity $F$ is said to be an extensive variable iff $F$ is Positively Homogeneous of degree one (PH1 in short), i.e.

$$
\forall \lambda \in \mathbb{R}^{+}, \forall W \in C, F(\lambda W)=\lambda F(W) .
$$


Definition 2. Let $F$ be an application from $C$ into $\mathbb{R}^{+}$. The quantity $F$ is said to be an intensive variable iff $F$ is Positively Homogeneous of degree zero (PHO in short), i.e.

$$
\forall \lambda \in \mathbb{R}^{+}, \forall W \in C, F(\lambda W)=F(W) .
$$

By taking the ratio of two components of $W$, it is possible to construct several intensive variables, either massic or volumic ones:

- the specific volume: $\tau=\frac{V}{M}$,

- the specific entropy: $s=\frac{S}{M}$,

- the density: $\rho=1 / \tau=\frac{M}{V}$,

- the volumic entropy: $\sigma=\rho s=\frac{S}{V}$.

Although it is usual to consider the system through its entropy function [HS06], we decide here to describe it by its internal energy $E$. The fundamental equation of a simple system is then of the form

$$
E:(M, S, V) \rightarrow E(M, S, V) .
$$

We enunciate here the main features of a fluid energy (for physical justifications, we refer to [Ca85]).

Postulat 1. The energy $E$ satisfies the following properties:

- $E$ is extensive.

- $E$ is convex with respect to $W=(M, S, V)$.

Assuming that $E$ is continuously differentiable we can define the temperature, the thermodynamical pressure and the chemical potential (or Gibbs potential) of the fluid

$$
\begin{aligned}
T & =\frac{\partial E}{\partial S}, \\
p & =-\frac{\partial E}{\partial V}, \\
\mu & =\frac{\partial E}{\partial M} .
\end{aligned}
$$

As gradients of PH1 functions, these functions are $\mathrm{PH} 0$ and thus intensive variables. We recover then the classical relation of thermodynamics in extensive variables

$$
d E=T d S-p d V+\mu d M .
$$

It is also possible to give an intensive form of the energy, either a massic form

$$
\epsilon(\tau, s)=E\left(1, \frac{S}{M}, \frac{V}{M}\right)=\frac{E}{M},
$$

or a volumic form

$$
\varepsilon(\rho, \sigma)=\rho \epsilon=\frac{E}{V} .
$$


From (6) it is easy to check that we also have

$$
d \epsilon=T d s-p d \tau .
$$

We have the following result

Proposition 1. The equation of state $\epsilon=\epsilon(\tau, s)$ is a convex function of $\tau$ and s. Moreover the volumic internal energy $\varepsilon=\varepsilon(\rho, \sigma)$ is also a convex function of $\rho$ and $\sigma$.

In the following, we will suppose that $\epsilon$ and $\varepsilon$ are $\mathcal{C}^{2}$ strictly convex functions for a pure phase, liquid or vapour. For a mixture, these functions will be merely $C^{1}$, see Section 1.2.

1.2. Immiscible mixtures. We consider now two phases of the same pure body. Each phase is characterized by its own energy function $E_{i}, i=1,2$, depending on the state $W_{i} \in C$. The extensive variables for the whole system are

$$
\begin{aligned}
M & =M_{1}+M_{2}, \\
S & =S_{1}+S_{2} .
\end{aligned}
$$

If the two phases are immiscible, we also have

$$
V=V_{1}+V_{2} .
$$

In thermodynamics, it is usual to express the entropy maximum principle. This principle is equivalent to the energy minimum principle. In this paper, we chose the later, only because it is a common habit in mathematics to solve minimization problems instead of maximization problems.

Postulat 2. Out of equilibrium, the energy of the system reads

$$
E\left(W_{1}, W_{2}\right)=E_{1}\left(W_{1}\right)+E_{2}\left(W_{2}\right) .
$$

At equilibrium, the energy of the system tends to decrease down to a minimum value, i.e.

$$
\begin{gathered}
E(W)=\min _{\left(W_{1}, W_{2}\right) \in \Omega} E\left(W_{1}, W_{2}\right) \\
\text { with }\left(W_{1}, W_{2}\right) \in \Omega \Longleftrightarrow\left\{\begin{array}{l}
W_{1}=\left(M_{1}, S_{1}, V_{1}\right) \in C, \\
W_{2}=\left(M_{2}, S_{2}, V_{2}\right) \in C, \\
M_{1}+M_{2}=M, \\
S_{1}+S_{2}=S, \\
V_{1}+V_{2}=V .
\end{array}\right.
\end{gathered}
$$

Because the set of constraints $\Omega$ is compact and the energy $\left(W_{1}, W_{2}\right) \mapsto$ $E\left(W_{1}, W_{2}\right)$ is smooth, the convex optimization problem has at least one solution. The convexity ensures that the value of the minimum is unique (even if the minimizer is not unique) and thus $W \mapsto E(W)$ is well defined. The minimization problem can be summed up by

$$
\begin{array}{r}
E(W)=\min _{W_{1} \in Q} E_{1}\left(W_{1}\right)+E_{2}\left(W-W_{1}\right) \\
\text { with } W_{1}=\left(M_{1}, S_{1}, V_{1}\right) \in Q \Longleftrightarrow\left\{\begin{array}{l}
0 \leq M_{1} \leq M, \\
0 \leq S_{1} \leq S, \\
0 \leq V_{1} \leq V .
\end{array}\right.
\end{array}
$$


If we assume that outside the cone $C$ the energies are extended by $+\infty$

$$
E_{i}(W)= \begin{cases}E_{i}(W) & \text { if } W \in C \\ +\infty & \text { elsewhere }\end{cases}
$$

Then the equilibrium energy is defined by

$$
E(W)=E_{1} \square E_{2}(W)=\min _{W_{1} \in \mathbb{R}^{3}}\left(E_{1}\left(W_{1}\right)+E_{2}\left(W-W_{1}\right)\right),
$$

where the symbol $\square$ stands for the inf-convolution operation.

The inf-convolution operation possesses many properties

- it ensures the existence and the convexity of the equilibrium energy E,

- it is closely related to the Legendre-Fenchel operation.

Some basic definitions and properties about these two operations are developed in Section 2.1. Moreover fast algorithms exist to compute infconvolution and Legendre-Fenchel operations. Such an algorithm is detailed in Section 2.3 and is applied to the computation of approximate equilibrium pressure laws when analytical expression are not available (see Section 3).

The optimization process can also be written with intensive variables. In this case, there is a nice a geometrical interpretation of the intensive mixture energy (see for instance [Ca85], [Ja01],[AFK07]).

Theorem 1. At equilibrium the mixture energy $\epsilon$ is the closed convex hull of the minimum of the two energies $\epsilon_{1}$ and $\epsilon_{2}$. In other words

$$
\epsilon=\operatorname{co}\left(\min \left(\epsilon_{1}, \epsilon_{2}\right)\right) \text {. }
$$

This theorem is proved in [AFK07] with geometric arguments.

Remark 2. In section 2 we provide another proof based on the properties of the inf-convolution and the Legendre transform.

In the sequel we use the following notation

Definition 3. Considering two phases of energies $\epsilon_{i}, i=1,2$, the immiscible mixture energy is denoted

$$
\epsilon_{1} \mid \epsilon_{2}:=\operatorname{co}\left(\min \left(\epsilon_{1}, \epsilon_{2}\right)\right)
$$

It would be convenient to deal with the convex change of variables

$$
\varepsilon_{i}\left(\rho_{i}, \sigma_{i}\right) \rightarrow \epsilon_{i}\left(\tau_{i}, s_{i}\right), i=1,2 .
$$

such that $\rho_{i}=\frac{1}{\tau_{i}}, \sigma_{i}=\rho_{i} s_{i}$. The theorem (1) is also true for the volumic energy.

Theorem 2. At equilibrium the surface of the mixture energy $\varepsilon$ is the closed convex hull of the surfaces $\varepsilon_{1}$ and $\varepsilon_{2}$, that is

$$
\varepsilon=\operatorname{co}\left(\min \left(\varepsilon_{1}, \varepsilon_{2}\right)\right)=\varepsilon_{1} \mid \varepsilon_{2} .
$$


1.3. Miscible mixtures. We consider now two phases of a same pure body, totally described by their energies $E_{i}, i=1,2$. As in the immiscible case each phase is characterized by its vector $W_{i}$ of parameters $W_{i}=\left(M_{i}, S_{i}, V_{i}\right) \in C$. Because the two fluids are perfectly miscible, they can occupy the whole volume. The set of constraints $\Omega$ is thus slightly changed

$$
\left(W_{1}, W_{2}\right) \in \Omega \Longleftrightarrow\left\{\begin{array}{l}
\left(M_{1}, V_{1}, S_{1}\right) \in C, \\
\left(M_{2}, V_{2}, S_{2}\right) \in C, \\
M_{1}+M_{2}=M, \\
S_{1}+S_{2}=S, \\
V_{1} \leq V, \\
V_{2} \leq V .
\end{array}\right.
$$

In this section we express the energy of such a mixture in terms of infconvolution both for extensive and intensive variables. The minimal energy will be reached at pressure equilibrium. The derivatives of $E$ with respect to $V_{1}$ and $V_{2}$ are respectively $p_{1}$ and $p_{2}$. As a result, assuming that the pressures are positive, the minimum will be obviously attained for the saturated constraint

$$
V_{1}=V_{2}=V .
$$

Therefore the equilibrium mixture energy is obtained by minimizing the following expression

$$
E\left(W_{1}, W_{2}\right)=E_{1}\left(M_{1}, S_{1}, V\right)+E_{2}\left(M_{2}, S_{2}, V\right) .
$$

Eliminating $W_{2}$, the mixture energy is again an inf-convolution of $E_{1}$ and $E_{2}$ but only in the mass and entropy variables.

Definition 4. Considering $Z_{1}=\left(M_{1}, S_{1}\right) \in Q$ with

$$
Q=\left\{\left(M_{i}, S_{i}\right), 0 \leq M_{i} \leq M, 0 \leq S_{i} \leq S\right\},
$$

we extend the energies $E_{i}$ such that

$$
E_{i}(Z, V)= \begin{cases}E_{i}(Z, V) & \text { if } Z \geq 0, \\ +\infty & \text { elsewhere. }\end{cases}
$$

The equilibrium energy reads thus

$$
\begin{aligned}
E(Z, V) & =\left(E_{1} \square E_{2}\right)(Z, V) \\
& =\min _{Z_{1} \in Q} E_{1}\left(Z_{1}, V\right)+E_{2}\left(Z-Z_{1}, V\right) .
\end{aligned}
$$

Using the fact that $E_{i}$ are PH1 functions, we can define an intensive formulation

Theorem 3. The volumic energy of a miscible mixture is given by an infconvolution operation

$$
\begin{aligned}
\varepsilon(\rho, \sigma) & =\varepsilon_{1} \square \varepsilon_{2}(\rho, \sigma) \\
& =\min _{\left(\rho_{1}, \sigma_{1}\right) \in D} \varepsilon_{1}\left(\rho_{1}, \sigma_{1}\right)+\varepsilon_{2}\left(\rho-\rho_{1}, \sigma-\sigma_{1}\right)
\end{aligned}
$$

with, for $i=1,2$,

$$
\begin{aligned}
& \sigma_{i}=\rho_{i} s_{i}, \\
& D=\left\{\left(\rho_{i}, \sigma_{i}\right), 0 \leq \rho_{i} \leq \rho, 0 \leq \sigma_{i} \leq \sigma\right\} \\
& \varepsilon_{i}\left(\rho_{i}, \sigma_{i}\right)=\epsilon_{i}\left(\tau_{i}, s_{i}\right) .
\end{aligned}
$$


Proof. We consider the mixture energy in extensive variables given by formula (26). Because it is a PH1 function, we can divide by the fixed volume $V$ to get

$$
\begin{aligned}
& E\left(\frac{M}{V}, \frac{S}{V}, 1\right)= \\
& \min _{\left(M_{1}, S_{1}\right) \in Q} E_{1}\left(\frac{M_{1}}{V}, \frac{S_{1}}{V}, 1\right)+E_{2}\left(\frac{M-M_{1}}{V}, \frac{S-S_{1}}{V}, 1\right) .
\end{aligned}
$$

Using the notations (29), the intensive formulation is straightforward.

\section{FASt LEGENDRE TRANSFORM}

2.1. Basic definitions and properties. In this section we recall basic facts about Legendre transform (LT) and we provide an efficient algorithm to compute Fast Legendre Transform (FLT). The Legendre transform is a useful operation in thermodynamics. Besides it takes place in the mixtures described in Section 1. Let us consider two fluids of volumic energies $\varepsilon_{i}$, $i=1,2$, then,

- if the two phases are immiscible, the mixture energy is the infconvolution of the two energies

$$
\varepsilon=\varepsilon_{1} \square \varepsilon_{2},
$$

- if the mixture is immiscible, the global energy is the convex hull of the minimum of the two energies

$$
\varepsilon=\varepsilon_{1} \mid \varepsilon_{2} .
$$

Applying twice the LT algorithm to any regular function turns out to give the convex hull of the function. To begin we sum up basic ideas and properties of Legendre transform. For more details we refer for instance to Hiriart-Urruty and Lemaréchal [HL01].

Definition 5. Let $f: \mathbb{R}^{n} \rightarrow \mathbb{R} \cup\{+\infty\}$ a (non necessarily convex) function such that

- $\exists x \in \mathbb{R}^{n}, f(x) \not \equiv+\infty$ (then $f$ is said to be proper),

- there exists an affine function minimizing $f$ on $\mathbb{R}^{n}$.

Then $f$ is said to be an admissible function.

It implies that $f(x)>-\infty, \forall x \in \mathbb{R}^{n}$, and in particular, if $f$ is a convex function, there is always an affine function minimizing $f$.

Definition 6. Let $f: \mathbb{R}^{n} \rightarrow \mathbb{R} \cup\{+\infty\}$ be an admissible function. Its conjugate $f^{*}$ is given by

$$
\begin{aligned}
f^{*}: s \in \mathbb{R}^{n} & \rightarrow \mathbb{R} \\
s & \mapsto \sup _{x \in \mathbb{R}^{n}}\{<s, x>-f(x)\} .
\end{aligned}
$$

were $\left\langle\cdot, \cdot>\right.$ denotes the scalar product on $\mathbb{R}^{n}$. The map $f \mapsto f^{*}$ is called the Legendre transform operation.

We can notice that $\forall s \in \mathbb{R}^{n}, f^{*}(s)>-\infty$ and that $f^{*}$ is an admissible function. In other words the domain of $f^{*}$ is the set of slopes of all the affine functions minimizing $f$ on $\mathbb{R}^{n}$, i.e. each $s \in \mathbb{R}^{n}$ is the slope of an affine map smaller than $f^{*}$. 
Proposition 2. Let $f$ be an admissible function. Then the conjugate $f^{*}$ is a lower semi-continuous everywhere (or closed) convex function.

Let us provide two examples using piecewise linear functions.

Example 1. We consider $\mathbb{I}_{[a, b]}$ the convex indicator function of the interval $[a, b] \subset \mathbb{R}$

$$
\mathbb{I}_{[a, b]}(x)= \begin{cases}0 & \text { if } a \leq x \leq b, \\ +\infty, & \text { elsewhere. }\end{cases}
$$

Then

$$
\left(\mathbb{I}_{[a, b]}\right)^{*}(\chi)=\sup _{x \in \mathbb{R}}\left(\chi x-\mathbb{I}_{[a, b]}(x)\right)=\sup _{x \in[a, b]} \chi x .
$$

In other words

$$
\left(\mathbb{I}_{[a, b]}\right)^{*}(\chi)=\left\{\begin{array}{cl}
0, & \text { if } \chi=0, \\
a \chi, & \text { if } \chi<0, \\
b \chi, & \text { if } \chi>0 .
\end{array}\right.
$$

Example 2. Let us consider the absolute value function on a certain domain $[a, b](a<0<b)$

$$
f(x)= \begin{cases}-x, & \text { if } a \leq x \leq 0, \\ x, & \text { if } 0 \leq x \leq b, \\ +\infty, & \text { elsewhere. }\end{cases}
$$

Then $f^{*}$ is the zero-function on $[-1,1]$ i.e.

$$
f^{*}(s)= \begin{cases}a s-1, & \text { if } s \leq-1, \\ 0, & \text { if }-1 \leq s \leq 1, \\ b s-1, & \text { if } s \geq 1 .\end{cases}
$$

Following the definition some properties are straightforward

Proposition 3. Let $f$ be an admissible function. Then

- $\forall t>0$, the conjugate of $g(x)=t f(x)$ is $g^{*}(s)=t f^{*}(s / t)$.

- $\forall t \neq 0$, the conjugate of $g(x)=f(t x)$ is $g^{*}(s)=f^{*}(s / t)$.

- We denote $f^{* *}$ the biconjugate of the function $f$ $\forall x \in \mathbb{R}^{n}, f^{* *}(x)=\left(f^{*}\right)^{*}(s)=\sup \left\{\left\langle s, x>-f^{*}(s)\right| s \in \mathbb{R}^{n}\right\}$.

The biconjugate $f^{* *}$ defines the closed convex hull of $f: \operatorname{cof}$.

In order to compute the mixture energy in the immiscible case, the LT operation can thus be applied twice.

According to the following result, the inf-convolution operation that defines the energy of a miscible mixture can be expressed in terms of Legendre transform too.

Proposition 4. Let $f_{1}$ and $f_{2}$ be two admissible functions. Then the Legendre transform of their inf-convolution (see Definition 5) is

$$
\left(f_{1} \square f_{2}\right)^{*}=f_{1}^{*}+f_{2}^{*},
$$

and conversely:

Proposition 5. Let $f_{1}$ and $f_{2}$ be two admissible functions. The conjugate of their sum is

$$
\left(f_{1}+f_{2}\right)^{*}=f_{1}^{*} \square f_{2}^{*} .
$$


The Legendre transform being an involutive operation for convex lsc functions, it ensures that if $f_{1}$ and $f_{2}$ are convex lsc

$$
\left(f_{1} \square f_{2}\right)=\left(f_{1}^{*}+f_{2}^{*}\right)^{*} .
$$

Proposition 6. Let $f_{1}$ and $f_{2}$ be two admissible functions. The conjugate of their min is the max of their conjugates, that is

$$
\left(\min \left(f_{1}, f_{2}\right)\right)^{*}=\left(\operatorname{co}\left(\min \left(f_{1}, f_{2}\right)\right)\right)^{*}=\max \left(f_{1}^{*}, f_{2}^{*}\right) .
$$

Thanks to these properties we provide another proof of Theorem 1.

Proof of theorem 1. We consider a immiscible mixture of two phases described by their extensive energies $E_{i}, i=1,2$. Because the Legendre transform is an involutive operation, it satisfies

$$
E^{* *}=\left(E_{1}^{*}+E_{2}^{*}\right)^{*}
$$

The Legendre transform of $E_{1}$ reads

$$
\begin{aligned}
E_{1}^{*}\left(M^{\prime}, S^{\prime}, V^{\prime}\right) & =\sup _{M, S, V \geq 0}\left(M M^{\prime}+S S^{\prime}+V V^{\prime}-E_{1}(M, S, V)\right) \\
& =\sup _{M, S, V \geq 0} V\left(\rho M^{\prime}+\sigma S^{\prime}+V^{\prime}-\varepsilon_{1}(\rho, \sigma)\right) \\
& =\sup _{V \geq 0} V\left(V^{\prime}+\varepsilon_{1}^{*}\left(M^{\prime}, S^{\prime}\right)\right) .
\end{aligned}
$$

Defining the set $A:=\left\{M^{\prime}, S^{\prime}, V^{\prime}, \varepsilon_{1}^{*}\left(M^{\prime}, S^{\prime}\right) \geq-V^{\prime}\right\}$, we observe that $E_{1}^{*}$ is the indicator function of $A$. In the same way the Legendre transform $E_{2}^{*}$ is the indicator function of $B:=\left\{M^{\prime}, S^{\prime}, V^{\prime}, \varepsilon_{2}^{*}\left(M^{\prime}, S^{\prime}\right) \geq-V^{\prime}\right\}$. As a consequence $E^{*}=\mathbb{I}_{A \cap B}$, where $A \cap B:=\left\{M^{\prime}, S^{\prime}, V^{\prime}, \max \left(\varepsilon_{1}^{*}, \varepsilon_{2}^{*}\right)\left(M^{\prime}, S^{\prime}\right) \geq-V^{\prime}\right\}$. Using Proposition 6 , the set $A \cap B$ can be written

$$
A \cap B:=\left\{M, S, V,\left(\min \left(\varepsilon_{1}, \varepsilon_{2}\right)\right)^{*}(M, S) \geq-V\right\} .
$$

In other words $\varepsilon$ is the convex hull of $\min \left(\varepsilon_{1}, \varepsilon_{2}\right)$.

Before describing the FLT algorithm, we pay a special attention the computation of the conjugate of boxed piecewise linear functions. We provide simple benchmark examples and refer to convergence results given in [Lu96] [Lu98].

2.2. The Legendre transform of boxed piecewise linear functions. In [Lu96], the author proposes a fast algorithm to compute Legendre transform and gives some results on theoretical convergence. However the approximation is good only on a certain part of the studied domain, even in one-dimensional case. The author recommends to take into account a larger domain to obtain convergence. To avoid this handling, we propose a better treatment of the boundaries of the studied domain.

Let $f$ be a real-valued function given on a domain $[a, b] \subset \mathbb{R}$. We assume that the function is extended outside its domain as usual

$$
f(x)= \begin{cases}f(x), & \text { if } x \in[a, b], \\ +\infty, & \text { elsewhere }\end{cases}
$$


Such a function is said to be boxed. The LT computation requires to discretize the function, that is to consider a boxed piecewise affine function. We define thus the following sampling operator.

Definition 7. Let $\left\{x_{0}, x_{1}, \ldots, x_{N}\right\}$ be a set of $N+1$ distinct points in the interval $[a, b]$. We note $\Pi f$ the projection of $f$ such that

$$
\forall x \in \mathbb{R}, \Pi f(x)= \begin{cases}\frac{f\left(x_{i+1}\right)-f\left(x_{i}\right)}{x_{i+1}-x_{i}}\left(x-x_{i}\right)+f\left(x_{i}\right), & \text { if } x_{i} \leq x \leq x_{i+1}, \\ +\infty, & i \in\{0, \ldots, N-1\}, \\ \text { elsewhere. }\end{cases}
$$

The linear interpolation operator defines thus a boxed piecewise linear function.

According to the Definition 6, the domain of the conjugate $f^{*}$ is the set of slopes of all the affine functions minimizing $f$. The Legendre transform of a convex function $f$ is the function $f$ itself and in particular

$$
\operatorname{co}(f)^{*}=f^{*} .
$$

For computing the Legendre transform, we will first compute the discrete convex hull of $\Pi f$, that we denote $\operatorname{co}(\Pi f)$. We propose a simple algorithm to build it, others can be found for instance in [Lu98].

Let $P_{i}$ be the points of coordinates $\left(x_{i}, \Pi f\left(x_{i}\right)\right), i=0, \ldots N$. The discrete convex hull co $(\Pi f)$ is given by the set of points $P_{k}, k=0, \ldots, N^{*}\left(N^{*} \leq N\right)$, such that the slopes of $\operatorname{co}(\Pi f)$, between two consecutive points, form an increasing sequence. For instance, if $f$ is a concave function, the convex hull co( $\Pi f)$ is the set $\left\{P_{0}, P_{N}\right\}$. Before giving our algorithm, let us fix some notations.

We denote by $G$ the list $G:=\left\{P_{i}, i=0, \ldots, N\right\}$ and $|G|$ its cardinal. The slope between two points $P_{i}$ and $P_{k}$ of $G$ is

$$
S\left(P_{i}, P_{k}\right)=\frac{\Pi f\left(x_{i}\right)-\Pi f\left(x_{k}\right)}{x_{i}-x_{k}} .
$$

The convexification algorithm is the following:

\section{PROCEDURE: CO $(\mathrm{G})$}

variable remove : boolean.

REPEAT

remove $=$ false

DO $i=1, \ldots,|G|-1$

IF $S\left(P_{i-1}, P_{i}\right)<S\left(P_{i}, P_{i+1}\right)$ THEN

$G=G \backslash\left\{P_{i}\right\}$,

END IF

remove $=$ true.

END DO

WHILE $(|G|>2)$ AND (remove=true)

END PROCEDURE: CO

Remark 3. This algorithm is obviously non optimal because at each iteration we scan all the points $P_{i}$. Under slight modifications it is easy to obtain $O(N)$ algorithm (by not rescaning the already ordered slopes). 


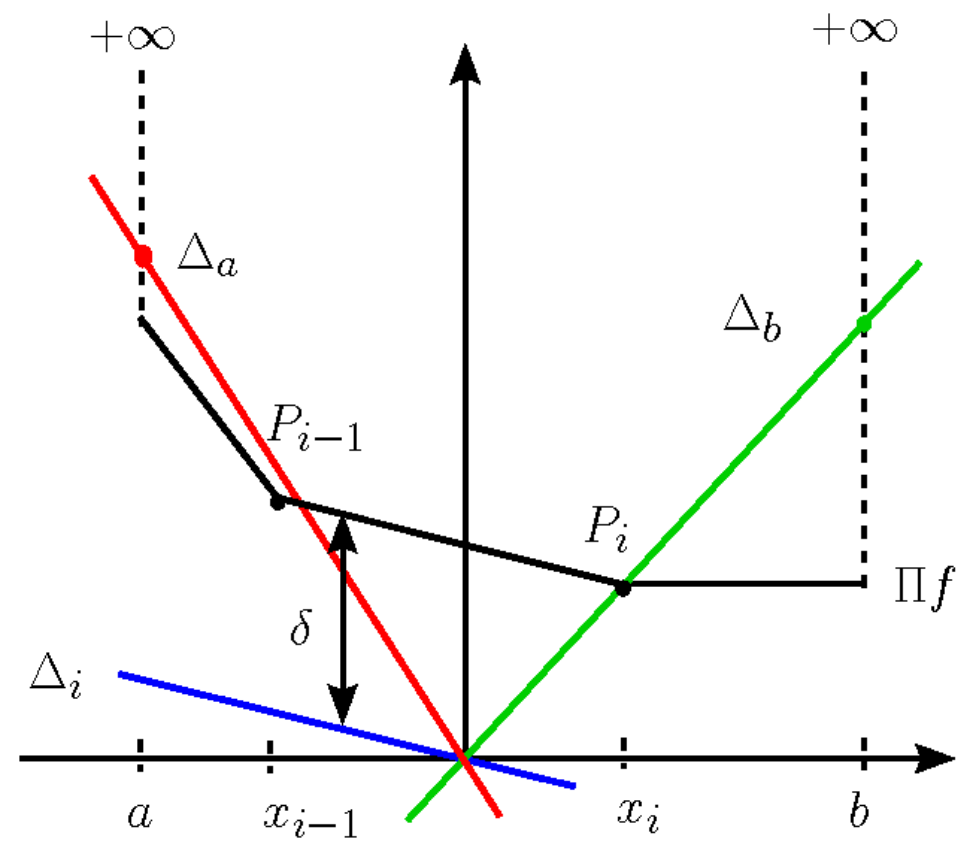

FiguRE 1. Legendre transform of a boxed piecewise linear function.

As a result $\operatorname{co}(\Pi f)$ is again a convex piecewise linear function, whose slopes

$$
\xi_{i}=\frac{\operatorname{co}(\Pi f)\left(x_{i}\right)-\operatorname{co}(\Pi f)\left(x_{i-1}\right)}{x_{i}-x_{i-1}}, i=1, \ldots, N^{*},
$$

where $N^{*}$ denotes the discretization of the dual domain of $\operatorname{co}(\Pi f)$. The sequence of slopes satisfy

$$
\xi_{1} \leq \xi_{2} \leq \ldots \leq \xi_{N^{*}} .
$$

Remark 4. In the sequel we set $N^{*}=N$ in order to avoid heavy notations. However the discretization of the dual domain can differ from the one of the real domain. 1)

The computation of the conjugate $(\operatorname{co}(\Pi f))^{*}$ is the following (see figure

- For any fixed $x \in] x_{i-1}, x_{i}\left[, i \in\{1, \ldots, N\}\right.$, let $\Delta_{i}$ be the linear line of slope $\xi_{i}$. By definition the value $(\operatorname{co}(\Pi f))^{*}\left(\xi_{i}\right)$ equals the maximum distance $\delta$ between the segment $\left[P_{i-1}, P_{i}\right]$ and $\Delta_{i}$.

- For any fixed $x<a$ (resp. $x>b$ ), we plot the affine line $\Delta_{a}$ of slope $\xi_{1}$ (resp. the affine line $\Delta_{b}$ of slope $\xi_{N}$ ). Because co( $(\Pi f)$ is extended by $+\infty$ outside $[a, b]$, the maximum distance between $\Delta_{a}$ (resp. $\Delta_{b}$ ) and $\operatorname{co}(\Pi f)$ is reached in $a$ (resp. $b$ ).

To sum up the conjugate function of $\pi^{*}$ reads

$$
(\operatorname{co}(\Pi f))^{*}(\xi)=\left\{\begin{array}{cl}
a \xi-\operatorname{co}(\Pi f)(a), & \text { if } \xi \leq \xi_{1}, \\
x_{i} \xi-\operatorname{co}(\Pi f)\left(x_{i}\right), & \text { if } \xi_{i} \leq \xi \leq \xi_{i+1}, i=1, \ldots N-1, \\
b \xi-\operatorname{co}(\Pi f)(b), & \text { if } \xi \geq \xi_{N}
\end{array}\right.
$$


The function $(\operatorname{co}(\Pi f))^{*}$ is a piecewise linear convex function defined on the dual domain $\left[\xi_{1}, \xi_{N}\right]$ and extended by lines of slopes $a$ and $b$ outside. In general the discretization points of $(\operatorname{co}(\Pi f))^{*}$ are no more equally spaced. It is necessary, for implementing the $2 \mathrm{D}$ algorithm described below, to sample the conjugate once again and to consider $\Pi\left((\operatorname{co}(\Pi f))^{*}\right)$. In order to avoid heavy notation, we again denote by $f^{*}$ the sampled conjugate $\Pi\left((\operatorname{co}(\Pi f))^{*}\right)$ and $f^{* *}$ the sampled biconjugate.

The same study can be performed to compute the biconjugate approximation $f^{* *}$. According to proposition $(2), f^{*}$ is a convex function. Its slopes, given by (37), read

$$
a=x_{0} \leq x_{1} \leq \ldots \leq x_{N-1} \leq x_{N}=b,
$$

up to sampling. It is easy to check that the domain of $f^{* *}$ is the exact initial domain of study $[a, b]$. Let us show the sampling effect on some examples.

Example 3. We consider the convex indicator $\mathbb{I}_{[-2,3]}$ (see Example 1). The computation of its conjugate and its biconjugate leads to the results of Figure 2. The computation regarding Example 2 are given in Figure 3.

Example 4. For $f(x)=x^{2}$ being given, the algorithm provides a discrete approximation of the biconjugate $f^{* *}$. The Figure 4 shows the effect of the discretization.

2.3. The Linear Legendre Transform algorithm. The Linear Legendre Transform algorithm (LLT) we propose is similar to the one given by [Br89] an [Lu98], except that we suppose real-valued functions to be extended by $+\infty$ outside their domain and conjugates are extended by affine maps.

Let us consider a function $f$ defined on $[a, b] \subset \mathbb{R}$. We introduce

$$
X=\left\{x_{0}, x_{1}, \ldots, x_{i}, \ldots, x_{N}\right\}
$$

a regular discretisation of $[a, b]$ such that

$$
x_{i}=a+i \frac{b-a}{N}, \quad i=0, \ldots, N .
$$

The discretization of $f$ is given by the definition (7), that is

$$
f \simeq \Pi f \text {. }
$$

We are now able to give the discrete one-dimensional LT algorithm:

Step 1: Compute the convex hull co( $\Pi f)$.

Step 2: Compute the discrete dual set $\Gamma$ of slopes of co( $\Pi f)$.

Step 3: Compute $(\operatorname{co}(\Pi f))^{*}$, according to formula (37).

Step 4: Compute $f^{*}=\Pi\left((\operatorname{co}(\Pi f))^{*}\right)$ using the linear interpolation operator (in order to recover $N$ discrete values).

Step 6: Extend $f^{*}$ by affine maps outside the dual domain $\Gamma$.

Although the two operations are identical, computationally speaking, let us detail the inverse Legendre transform algorithm.

Step 1: Compute the convex hull $\operatorname{co}\left(\Pi f^{*}\right)$.

Step 2: Compute $\Delta$, the discrete set of slopes of co( $\left.\Pi f^{*}\right)$.

Step 3: Compute $\left(\operatorname{co}\left(\Pi f^{*}\right)\right)^{*}$, according to formula (37). 

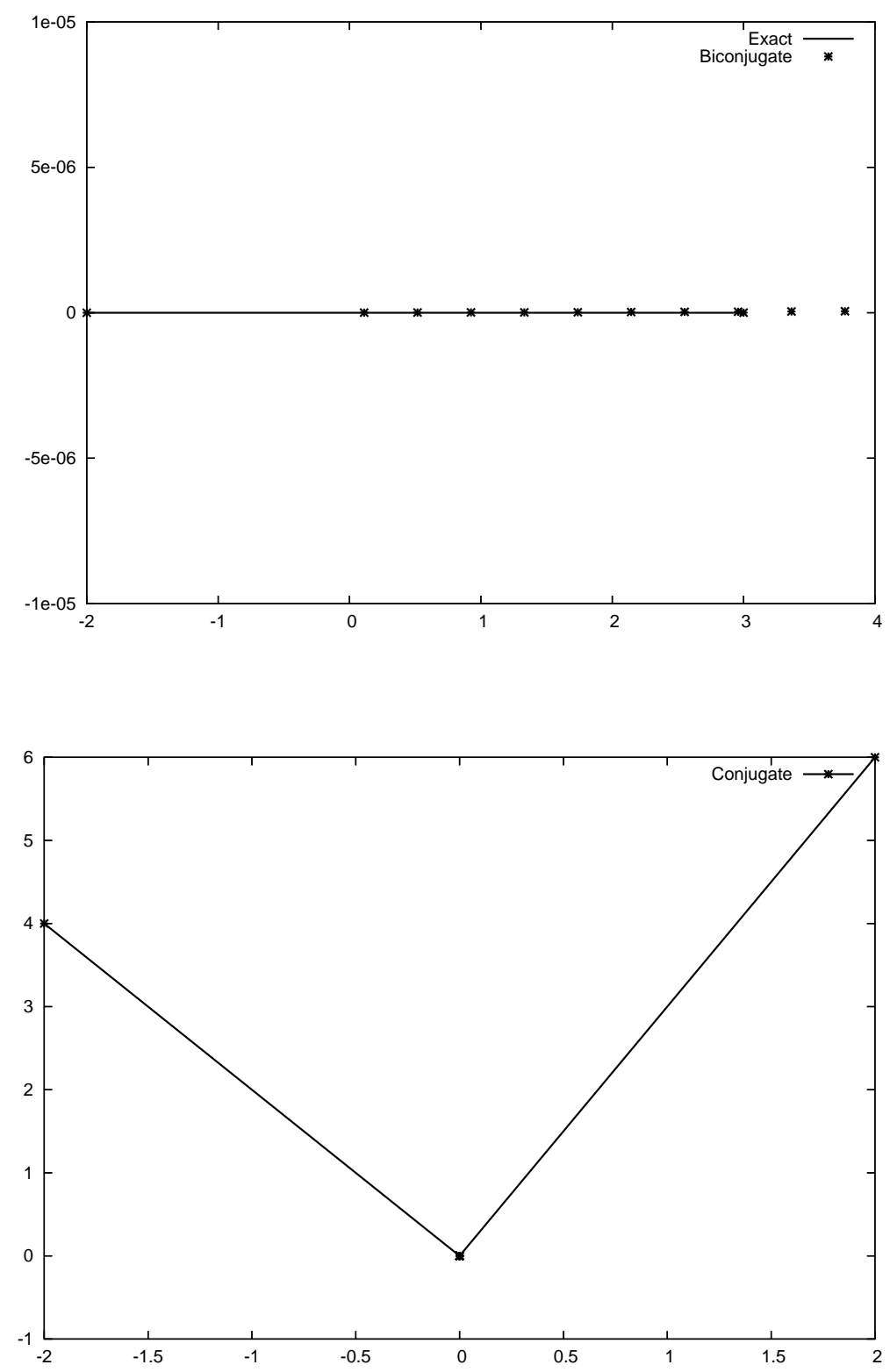

Figure 2. Conjugate and Biconjugate of $\mathbb{I}_{[-2,3]}$.

Step 5: Compute $f^{* *}=\Pi\left(\left(\operatorname{co}\left(\Pi f^{*}\right)\right)^{*}\right)$ using the linear interpolation operator (in order to recover $N$ discrete values).

Step 6: Extend $f^{* *}$ by $+\infty$ outside the dual domain $\Delta$.

By construction the set $\Delta$ turns to be the initial real domain $X$, up to sampling. 

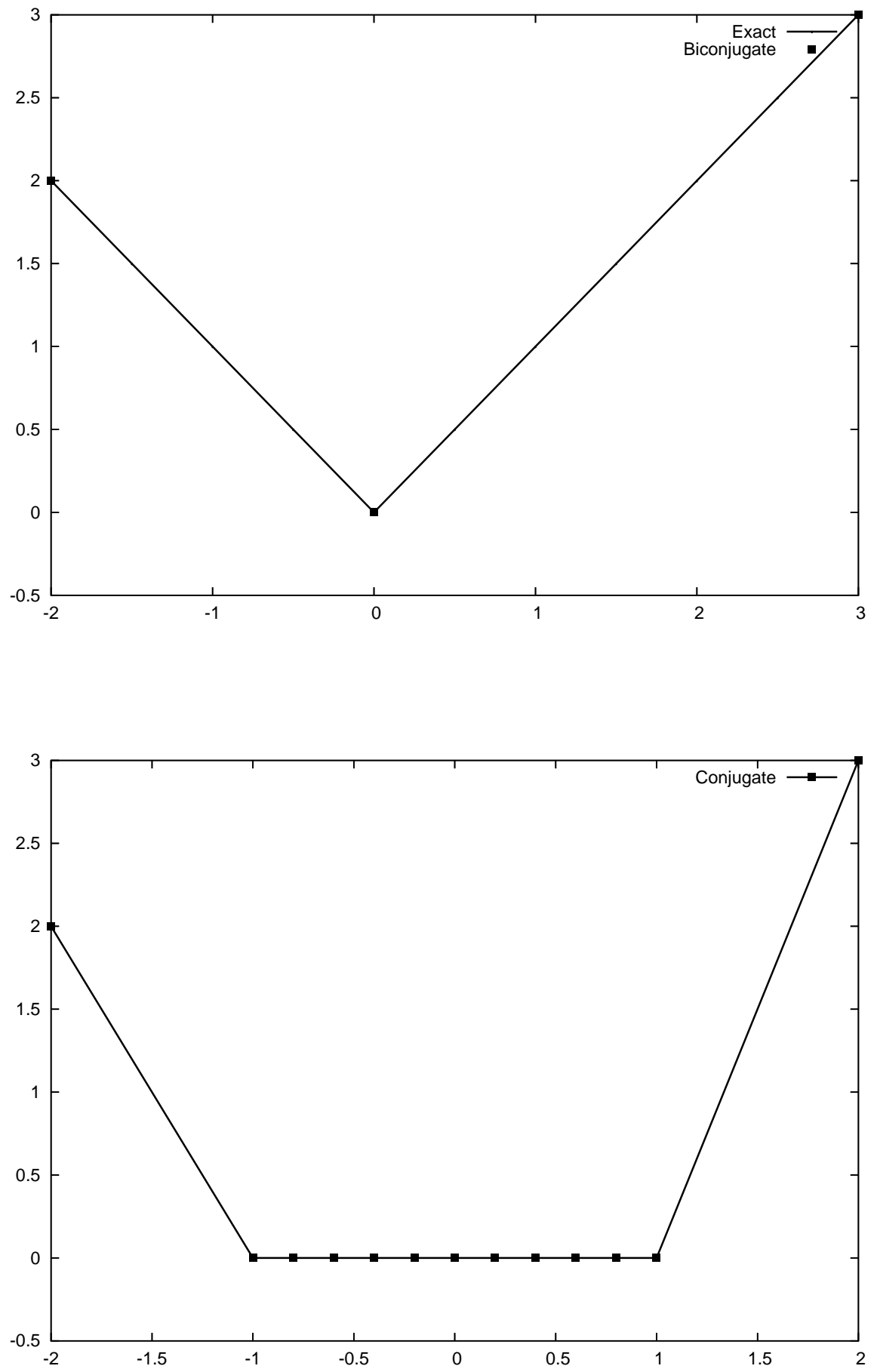

Figure 3. Conjugate and Biconjugate of the $f(x)=|x|$. 

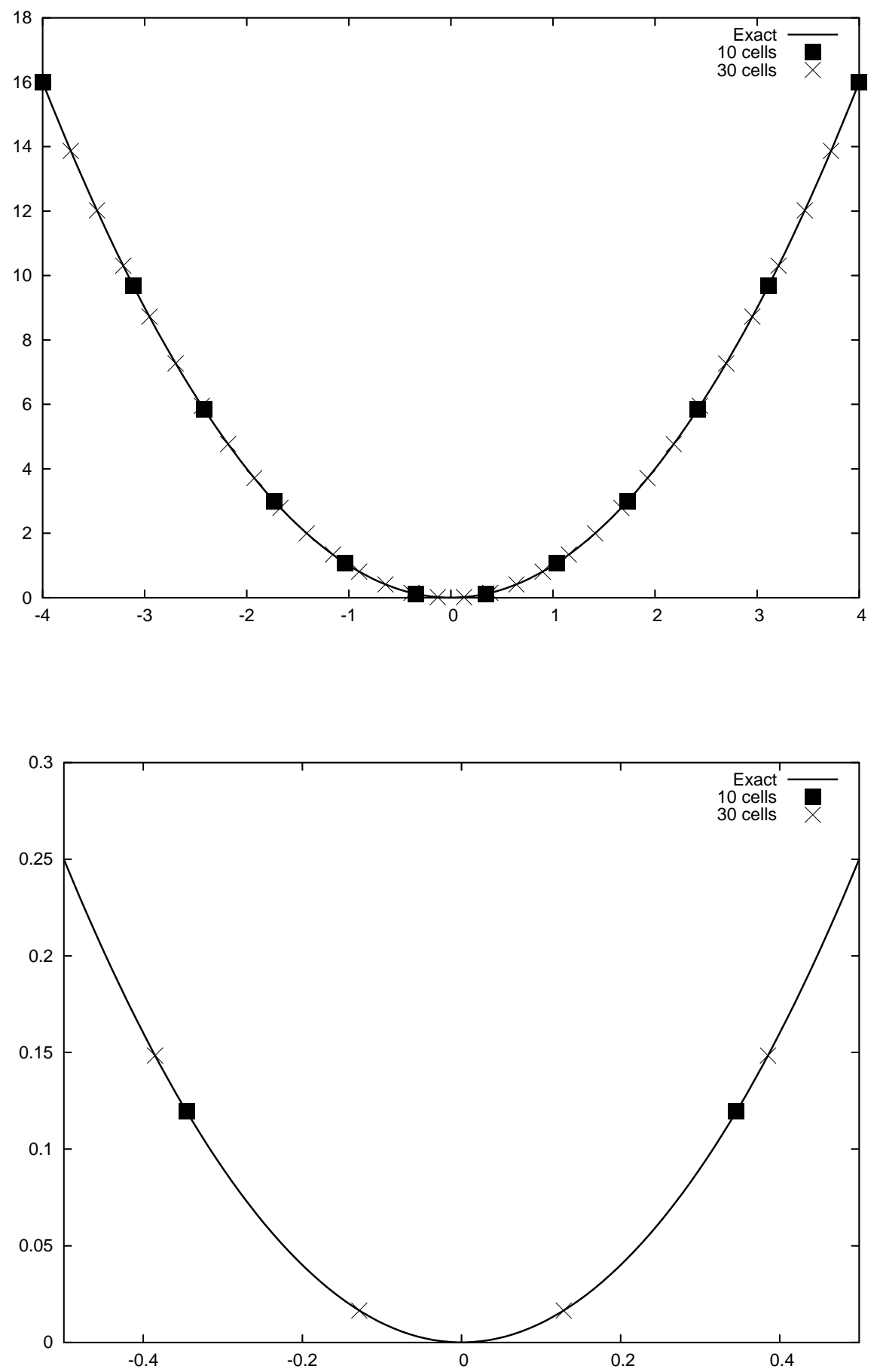

Figure 4. Conjugate and Biconjugate of the $f(x)=x^{2}$. 
2.4. Extension to higher dimensions. The extension to higher dimensions is rather straightforward, because the LT in $\mathbb{R}^{n}$ may be factorized in $n$ LT in $\mathbb{R}$. Moreover the planar algorithm has a $O(N)$ complexity.

For $f: \mathbb{R}^{n} \rightarrow \mathbb{R} \cup\{+\infty\}$, Definition 6 gives the expression of the conjugate $f^{*}$

$$
f^{*}(\xi)=\sup _{x_{1} \in \mathbb{R}}\left[\xi_{1} x_{1}+\sup _{x_{2} \in \mathbb{R}}\left[\xi_{2} x_{2}+\ldots+\sup _{x_{d} \in \mathbb{R}}\left[\xi_{d} x_{d}-f(x)\right]\right]\right] .
$$

For more clearness, let us detail the two-dimensional case

$$
f^{*}\left(\xi_{1}, \xi_{2}\right)=\sup _{x_{1} \in \mathbb{R}}\left[\xi_{1} x_{1}+\sup _{x_{2} \in \mathbb{R}}\left[\xi_{2} x_{2}-f\left(x_{1}, x_{2}\right)\right]\right] .
$$

In the sequel we will denote $f^{*, 1}$ (resp. $f^{*, 2}$ ) the partial Legendre transform with respect to the variable $x_{1}$ (resp. $x_{2}$ ). Then it is easy to prove that

Proposition 7. Only one-dimensional Legendre transforms are involved in the multidimensional problem, i.e.

$$
f^{*}=\left(-f^{*, 2}\right)^{*, 1}
$$

This proposition can easily be generalized to higher dimensions. Therefore convergence and complexity results enunciated in [Lu98] hold.

Remark 5. If $f$ is convex with respect to $x_{1}$, then $f^{*, 2}$ is a concave function of $x_{1}$. It is necessary to consider $-f^{*, 2}$ in order to recover a convex function.

A bivariate function $f:[a, b] \times[c, d] \rightarrow \mathbb{R}$ being given, the two-dimensional algorithm proceeds as follow:

\section{Step 1: discretization:}

(1) Compute the discrete sets $X$ and $Y$ such that:

$$
\begin{aligned}
& X:=\left\{x_{i}=a+i \frac{b-a}{N}, \quad i=0, \ldots, N\right\}, \\
& Y:=\left\{y_{j}=c+j \frac{d-c}{M}, \quad j=0, \ldots, M\right\} .
\end{aligned}
$$

Remark 6. In order to avoid heavy notations and without a loss of generality, we assume that the discretization of the dual domain is the same as the one of the real domain.

Step 2: For $y_{j} \in Y, j \in\{1, \ldots, M\}$

(1) Compute the piecewise affine function $\Pi f\left(\cdot, y_{j}\right)$.

(2) Compute the convex hull $\operatorname{co}(\Pi f)\left(\cdot, y_{j}\right)$.

(3) Compute the set $\Xi=\left\{\xi_{1}, \ldots, \xi_{N}\right\}$ of slopes of $\operatorname{co}(\Pi f)\left(\cdot, y_{j}\right)$.

(4) Compute $f^{*, 1}=\Pi\left((\operatorname{co}(\Pi f))^{*, 1}\right)$ using the one-dimensional LT algorithm.

Step 3: For $\xi_{i} \in \Gamma, i \in\{1, \ldots, N\}$ :

(1) Compute the piecewise affine function $\Pi\left(-f^{*, 1}\right)\left(\xi_{i}, \cdot\right)$.

(2) Compute the convex hull $\operatorname{co}\left(\Pi\left(-f^{*, 1}\right)\right)\left(\xi_{i}, \cdot\right)$.

(3) Compute the set $\Gamma=\left\{\gamma_{1}, \ldots, \gamma_{M}\right\}$ of slopes of $\operatorname{co}\left(\Pi\left(-f^{*, 1}\right)\right)\left(\xi_{i}, \cdot\right)$.

(4) Compute $f^{*}=\Pi\left(\left(\operatorname{co}\left(\Pi\left(-f^{*, 1}\right)\right)\right)^{*, 2}\right)$ using the one-dimensional LT algorithm. 
Applying this algorithm once again, we recover an approximation of the closed convex hull of the initial surface on the exact initial domain $[a, b] \times$ $[c, d]$, that is

$$
f^{* *} \simeq \operatorname{co}(f) .
$$

Remark 7. Let us consider $f: \mathbb{R}^{2} \rightarrow \mathbb{R}$. Its graph is said to be a ruled surface in the $\xi$ direction if

$$
f(\xi, \gamma)=g(\gamma)+h(\gamma)\left(\xi-\xi_{0}\right),
$$

for some functions $g$ and $h,(\xi, \gamma) \in \mathbb{R}^{2}$. According to this definition, for $\xi<\xi_{1}$ and $\xi>\xi_{N}$ (resp. for $\gamma<\gamma_{1}$ and $\gamma>\gamma_{M}$ ), the conjugate $f^{*, 1}(\xi, \gamma)$ (resp. $f^{*, 2}(\xi, \gamma)$ ) defines a ruled surface in the $\xi$ direction (resp. $\gamma$ direction). As a consequence the graph of $f^{*}$ is locally a hyperplane in $\mathbb{R}^{2} \backslash\{([a, b] \times \mathbb{R}) \cup(\mathbb{R} \times[c, d])\}$.

Remark 8. While $O(N)$ convex hull algorithms exist in the planar case, it is no more true in $\mathbb{R}^{n}, n \geq 3$. Following Proposition 3, using the LT operation twice is an efficient way to compute an approximate convex hull.

\section{Numerical APPLiCATiOnS TO THERMODYNAMiCS}

3.1. Numerical change of variables. In this section the multidimensional FLT algorithm is applied to some basic phase transition models, namely the van der Waals gas and the perfect gas mixture. Analytical expression of the mixture entropy are available in [Ja01], [AFK07] and [HS06]. It is classical to study the EOS through the isothermal curves in the $(\tau, p)$ plane. Using the Legendre transform algorithm, we provide a simple algorithm in order to compute the isothermal curves. We consider a fluid described by its energy $\epsilon(\tau, s)$. The Legendre transform of $\epsilon$ with respect to $s$, denoted $F$, is

$$
F(\tau, T)=\epsilon^{*, s}(\tau, T)=T s-\epsilon(\tau, s),
$$

where $T=\frac{\partial \epsilon}{\partial s}(\tau, s)$.

The function $F$ is nothing else than the opposite of the Helmoltz free energy function in intensive variables. Because

$$
d F=p d \tau+s d T,
$$

the pressure can be computed as

$$
\frac{\partial F}{\partial \tau}(\tau, T)=p(\tau, T) .
$$

We see that the isothermal curves can be constructed by applying once a FLT algorithm in the $s$ direction and then by computing the slopes in the $\tau$ direction.

3.2. Maxwell construction for a van der Waals gas. In the perfect gas model, the gas atoms are supposed to not interact and their volumes are negligible. For high densities, this model is no more adapted (the distance between particles is about the interaction distance). The van der Waals equation of state is an extension of the perfect gas law, which takes into 


\begin{tabular}{|c|c|}
\hline Constants & Value \\
\hline$R$ & $8.314 J \cdot \mathrm{K}^{-1} \cdot \mathrm{mol}^{-1}$ \\
$c_{V}$ & $4186 J . \mathrm{Kg}^{-1} \cdot \mathrm{K}^{-1}$ \\
$a$ & $0.544 \mathrm{~Pa}^{-6} \mathrm{~m}^{-6}$ \\
$b$ & $30.510^{-6} \mathrm{~m}^{3}$ \\
\hline
\end{tabular}

TABLE 1. van der Waals constants for liquid water

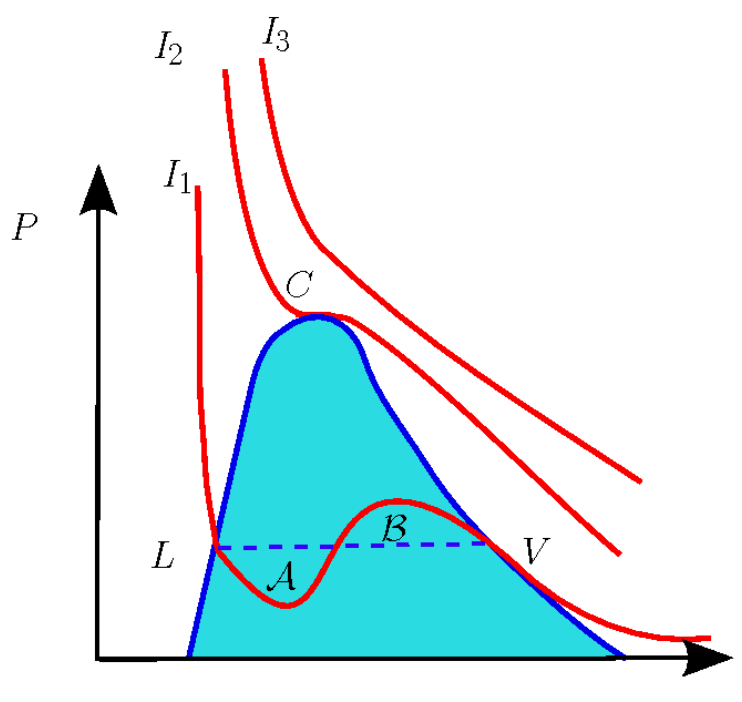

$\tau$

FiguRE 5. isothermal curves for the van der Waals EOS

account the attraction between atoms and the decrease of the free volume. The complete EOS is given by

$$
\epsilon(\tau, s)=(\tau-b)^{-R / c_{V}} \exp \left(\frac{s}{c_{V}}\right)-\frac{a}{\tau}
$$

(it is extended by $+\infty$ for $\tau \leq b$ or $s \leq 0$ ) where $R$ is the perfect gas constant, $c_{V}$ is the specific heat, $a$ and $b$ are positive constants. These constants are given in the table 3.2 in the case of liquid water. For other materials we refer to [Ca85] or [Ja01].

This EOS can be applied to liquids as well as gases. But it is not physically correct in the phase transition area. In the mixture zone, liquid and gas are present by a continuous change of liquid fraction. While isothermal curves are monotone above the critical point $C$ (see Figure 5 ), it is no more true in the mixture zone. We can observe two local extrema in this area, called spinodal points. The maximum corresponds to supersaturated vapour and the minimum to the overexpanded liquid. Between these two points $\frac{\partial p}{\partial \tau}(\tau, T)>0$, which is physically excluded. In order to get an admissible behaviour of phase transition in the mixture zone the equilibrium pressure must be calculated as a function of the temperature. This construction is called the Maxwell's principle. It consists in constructing the segment $[L, V]$ in such a way that the volume $\mathcal{A}$ is equal to the volume $\mathcal{B}$. Along the segment 


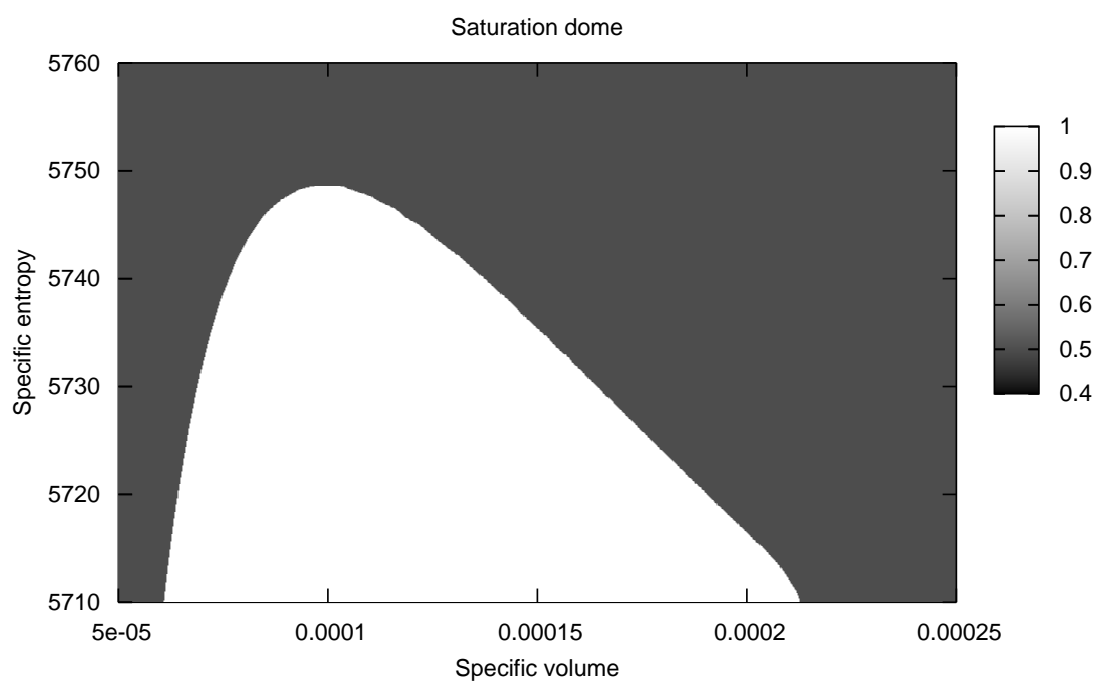

Figure 6. Saturation dome of the van der Waals model (before convexification)

$[L, V]$ (called Maxwell line) the two phases coexist at equilibrium. The main problem is that the positions of both points cannot be found analyticaly and requires a numerical resolution that can be CPU consumming when many EOS evaluations are needed. In fact the Maxwell contruction is equivalent to set equality of Gibbs potentials, pressures and temperatures along the segment $[L, V]$. In other words it is equivalent to the construction of the bitangent plan between two stable sticks of the van der Waals law [AFK07], i.e. to compute the convex hull of the energy surface. This can be achieved in a very simple way by applying twice the FLT algorithm.

We consider a fluid described by the equation of state (46) for liquid water. The saturation dome in the $(\tau, p)$ plane is merely obtained by computing

$$
\epsilon(\tau, s)-\operatorname{co}(\epsilon(\tau, s)) \text {. }
$$

Where this quantity is $>0$ corresponds to the saturation dome. It leads to the results of Figure 6 . Using the change of variables introduced in the previous section, the isothermal curves in the spinodal area are easily depicted before the convexification process (see Figure 7) and after (see Figure 8).

3.3. Immiscible mixture of two perfect gases. In this section we consider an immiscible mixture at equilibrium. The vapour and liquid phases 


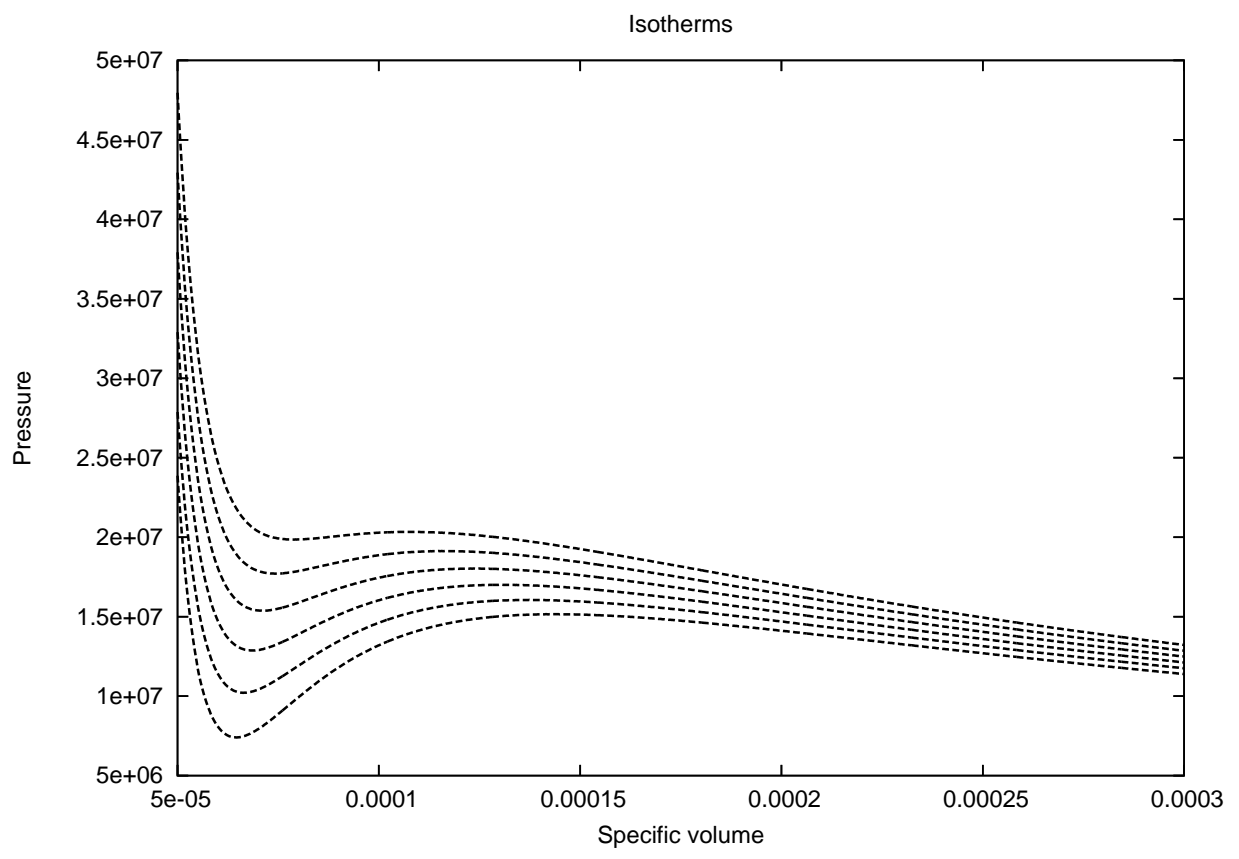

FiguRE 7. Isothermal curves of the van der Waals model (after convexification)

(denoted respectively 1 and 2) are modelled by a perfect gas laws

$$
\begin{aligned}
& \epsilon_{1}\left(s_{1}, \tau_{1}\right)=\exp \left(s_{1}\right) \tau_{1}^{1-\gamma_{1}}, \\
& \epsilon_{2}\left(s_{2}, \tau_{2}\right)=\exp \left(s_{2}\right) \tau_{2}^{1-\gamma_{2}},
\end{aligned}
$$

with $\gamma_{1}>\gamma_{2}$ (specific heats are supposed to be equal to 1 ). The optimization problem consists in fixing the Gibbs potentials, pressures and temperatures equilibrium. The equation of the coexistence curve in the $(T, p)$ plane follows

$$
p=\exp (-1)\left(\frac{\left(\gamma_{1}-1\right)^{\gamma_{1}-1}}{\left(\gamma_{2}-1\right)^{\gamma_{2}-1}}\right)^{\frac{1}{\gamma_{1}-\gamma_{2}}} T
$$

For detailed calculus we refer to [AFK07] and [Ja01]. The equation of the phase transition curve in the $(\tau, p)$ and $(\tau, \epsilon)$ is deduced from continuity of pressure and temperature between pure phases and mixture zone. Finally the global energy reads

(50) $\epsilon(\tau, s)= \begin{cases}\exp (s) \tau^{\left(1-\gamma_{1}\right)}, & \text { if } \quad \tau \leq \tau_{2}, \\ \exp \left(s-\left(1-\gamma_{1}\right)\left(\frac{\tau}{\tau_{1}}-1\right)\right) \tau_{1}^{1-\gamma_{1}}, & \text { if } \quad \tau_{2} \leq \tau \leq \tau_{1}, \\ \exp (s) \tau^{\left(1-\gamma_{2}\right)}, & \text { if } \quad \tau \geq \tau_{1} .\end{cases}$ 


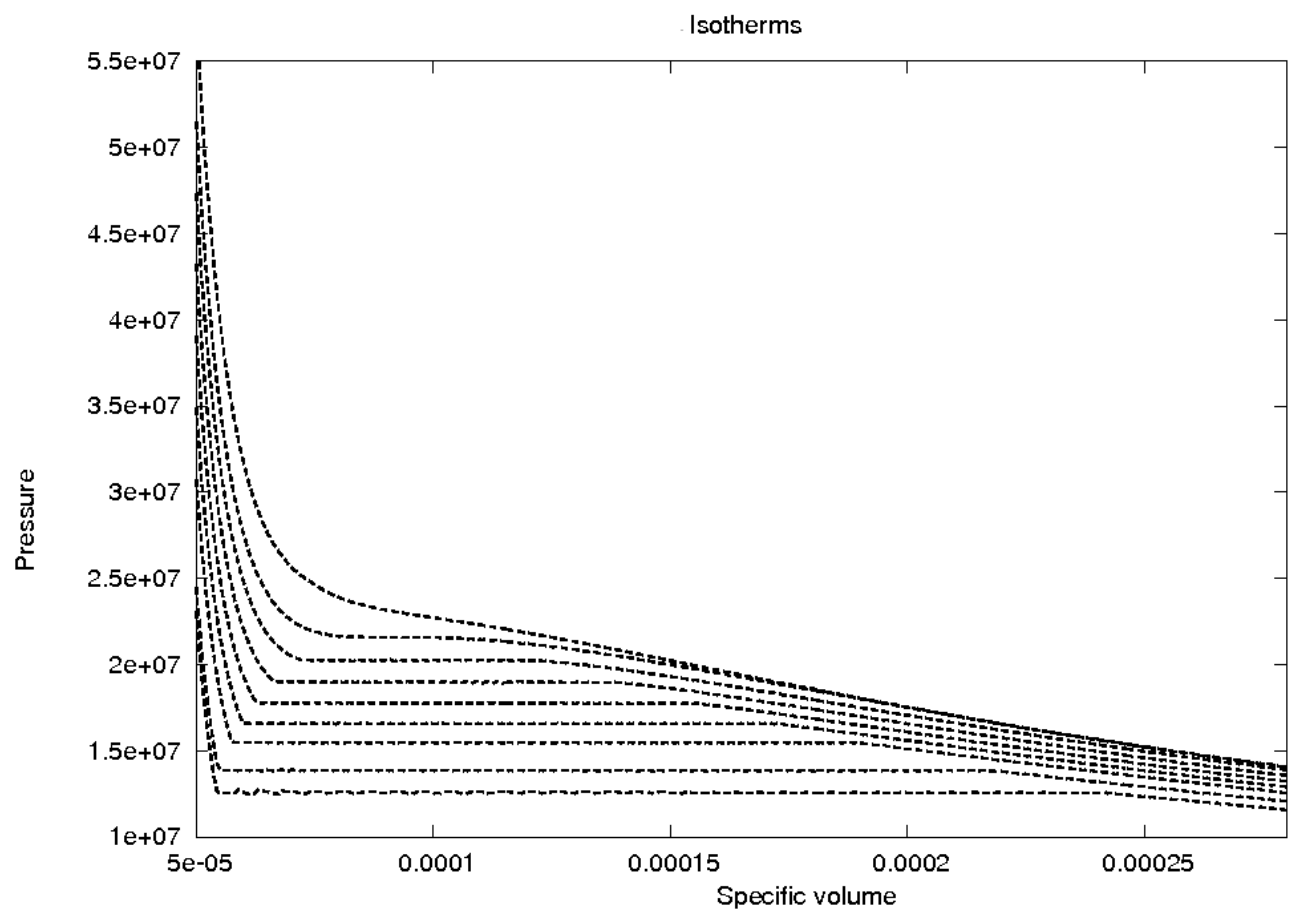

Figure 8. Isothermal curves of the van der Waals model
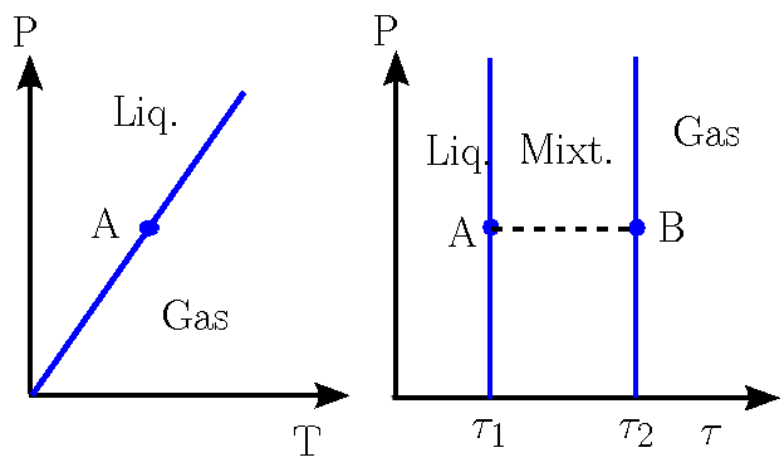

FiguRE 9. Isothermal curves for perfect gas mixture

Let $A$ be a point of the coexistence curve in the $(T, p)$ plane. It corresponds to a horizontal segment $A B$ in the $(\tau, p)$ plane (see figure 9 ). Contrary to the van der Waals EOS, the liquid vapour phase transition does not model a critical behaviour.

The same results results can be obtained numerically. Setting $\gamma_{1}=1.6$ and $\gamma_{2}=1.5$, we compute the saturation dome

$$
\left(\epsilon_{1} \mid \epsilon_{2}\right)-\min \left(\epsilon_{1}, \epsilon_{2}\right) .
$$




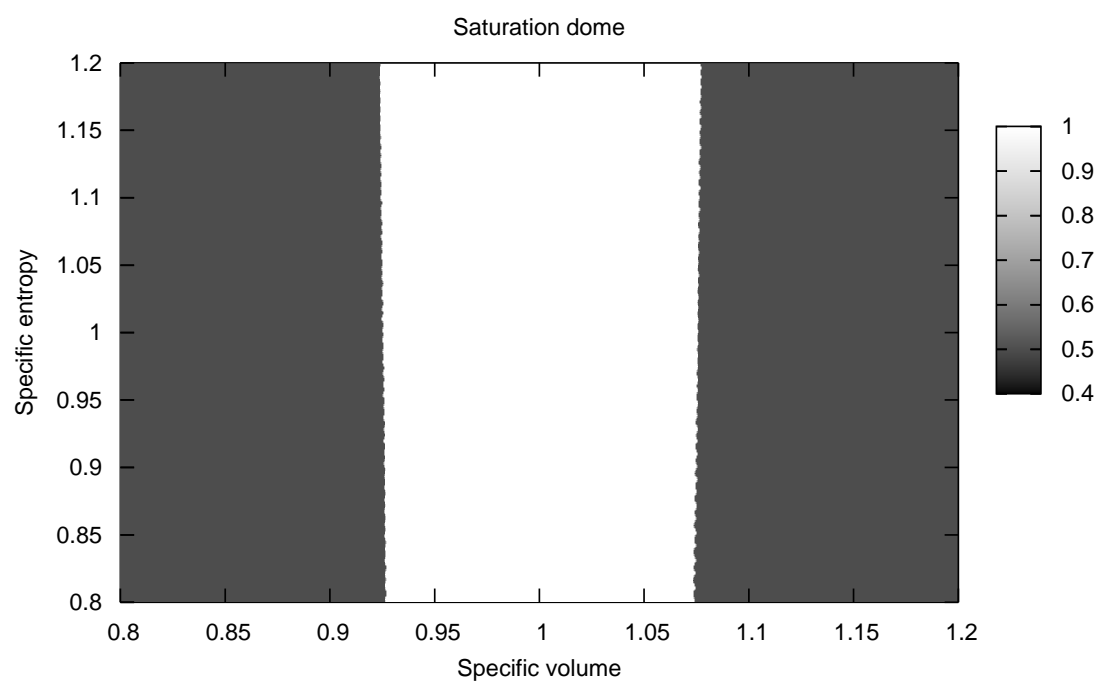

FIGURE 10. Saturation dome for perfect gas mixture.

We observe (see Figure 10) that the saturation dome does not present a critical point. Because the phase transition happens at constant pressure and temperature, isothermal curves curves are horizontal in the $(\tau, p)$ plane in the mixture zone. The isothermal curves present a kink at the saturation boundaries as shown on Figure 11.

3.4. Miscible mixture of two perfect gases. In Section 1 we constructed an algebraic structure of thermodynamic mixtures on the set of the convex functions equipped with the two binary operations $\mid$ and $\square$. According to Propositions 5 and 6 , the dual structure is a $(\max ,+)$ algebra on the Legendre space. We consider now a two phases mixture involving the infconvolution operation. The phases 1 and 2 are supposed to be miscible. The mixture is depicted by its intensive energy in terms of volumic variables $\varepsilon(\rho, \sigma)$.

Proposition 8. The Legendre transform of the energy $\varepsilon(\rho, \sigma)$ is the pressure of the mixture as a function of the temperature $T$ and the gibbs potential $\mu$ i.e.

$$
(\varepsilon(\rho, \sigma))^{*}=p(\mu, T) .
$$




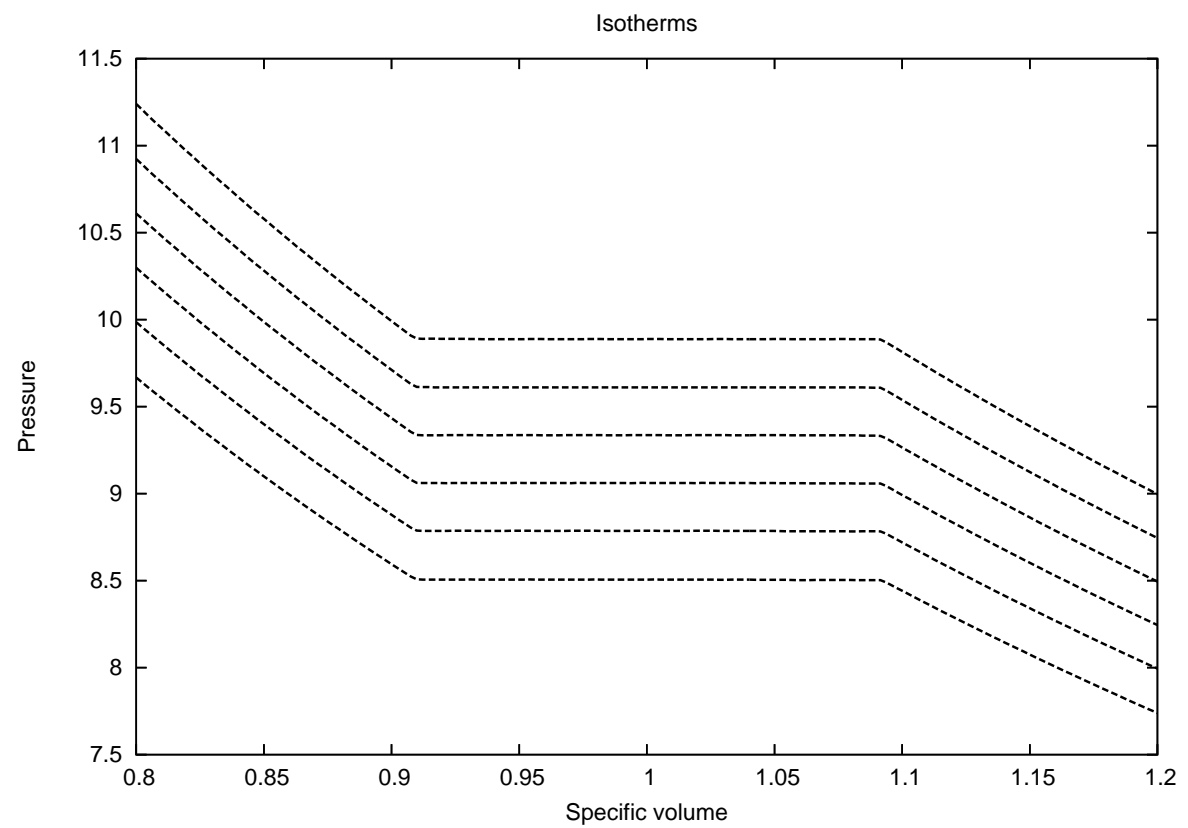

FIGURE 11. isothermal curves of perfect gas mixture

Proof. Because $\varepsilon=\rho \epsilon$, we can deduce that

$$
\begin{aligned}
d \varepsilon & =\rho d \epsilon+\epsilon d \rho \\
& =\rho\left(T d\left(\frac{\sigma}{\rho}\right)-p d\left(\frac{1}{\rho}\right)\right)+\epsilon d \rho \\
& =T d \sigma+\left(\frac{\varepsilon-\sigma T+p}{\rho}\right) d \rho \\
& =T d \sigma-\mu d \rho .
\end{aligned}
$$

In other words the partial derivatives of $\varepsilon$ are

$$
\begin{aligned}
& \frac{\partial \varepsilon}{\partial \sigma}=T, \\
& \frac{\partial \varepsilon}{\partial \rho}=-\mu .
\end{aligned}
$$

The Legendre transform of $\varepsilon$ reads thus

$$
\begin{aligned}
\varepsilon^{*}(-\mu, T) & =T \sigma-\mu \rho-\varepsilon \\
& =p(\mu, T) .
\end{aligned}
$$

Thus the pressure of the mixture can be directly computed in the Legendre space as the sum of each pressure

$$
\left(\varepsilon_{1} \square \varepsilon_{2}\right)^{*}=p_{1}+p_{2} .
$$


We recover the Dalton law.

Remark 9. Proposition 8 is very simple but also very important. It is another justification of working with volumic energy $\varepsilon$ instead of massic energy $\epsilon$. In the Legendre space, the pressure of a miscible mixture satisfies

$$
p(\mu, T)=p_{1}(\mu, T)+p_{2}(\mu, T)
$$

while the pressure of an immiscible mixture is given by

$$
p(\mu, T)=\max \left(p_{1}(\mu, T), p_{2}(\mu, T)\right) .
$$

We see that the natural mathematical framework for studying thermodynamics mixture is the $(\max ,+)$ algebra.

Remark 10. From physics, we know that the supercritical fluid is miscible with the vapor and the liquid phases. Let us consider a mixture of vapor (1), liquid (2) and supercritical phase (s). According to our theory, the pressure of the liquid/supercritical mixture should be

$$
p_{2}+p_{s}
$$

while the pressure of the vapor/supercritical mixture should be

$$
p_{1}+p_{s} .
$$

The pressure of the whole mixture is

$$
p=\max \left(p_{1}+p_{s}, p_{2}+p_{s}\right) .
$$

Using the distributivity of + with respect to max, we also have

$$
p=p_{s}+\max \left(p_{1}, p_{2}\right) .
$$

This formula is an interesting starting point for constructing supercritical models (richer than the van der Waals model) only from macroscopic thermodynamics arguments.

In a miscible mixture, when the temperature changes, the mass fraction of each phase is not constant (unless if we consider a miscible mixture of a gas with itself). In order to test numerically our construction, we consider a miscible mixture of two perfect gases and plot the isothermal curves as well as the mass fraction dependance on the temperature and the pressure. The isothermal curves are obtained by computing the derivative of the pressure $p$ with respect to the variable $\mu$ at fixed temperature $T$.

The Figure 12 presents the isotherms in the $(\tau, p)$ plane with $\mu \in[-10,-5]$ and $T \in[2,20]$. The polytropic exponents are $\gamma_{1}=1.4$ and $\gamma_{2}=1.8$. The isothermal curves are convex and are very similar to the perfect gas isotherms. The mass fraction profile is given in Figure 13. The quantity $\varphi=M_{1} / M$ increases with respect to the pressure. We check numerically that $0 \leq \varphi \leq 1$. We also have checked that if $\gamma_{1}=\gamma_{2}$ then the fraction $\varphi$ is constant and equals $1 / 2$. 


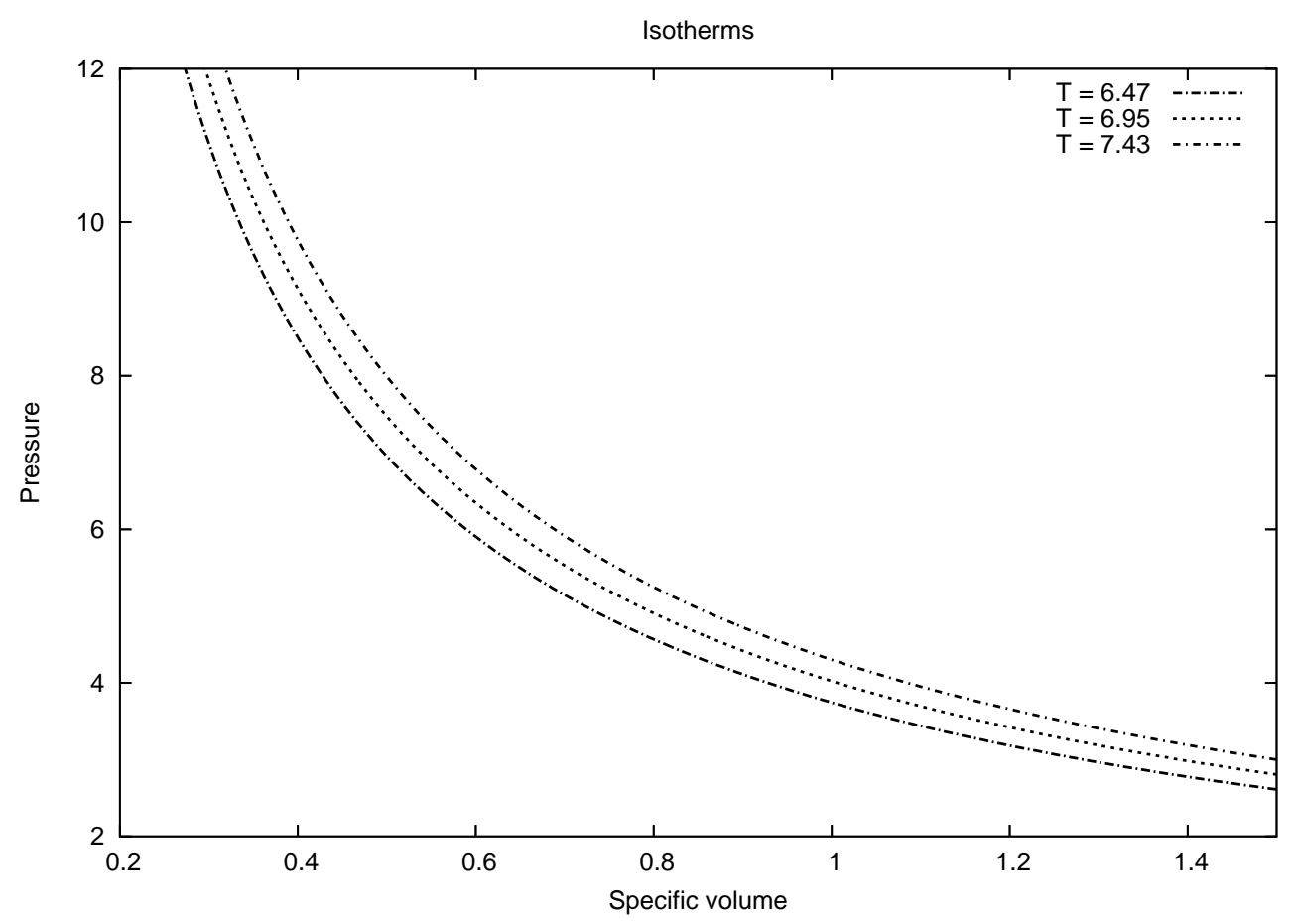

FiguRE 12. Isotherms of the miscible mixture

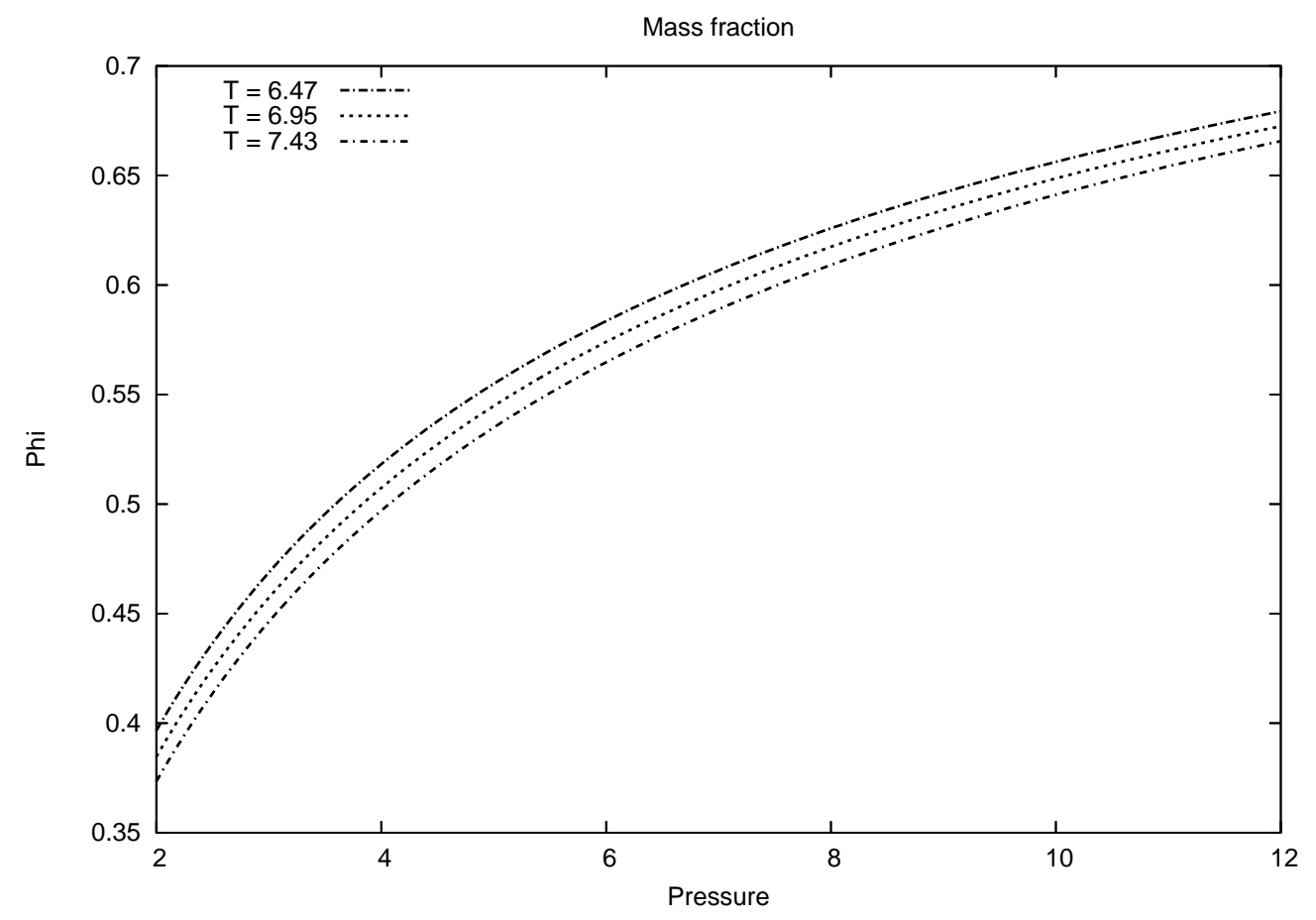

Figure 13. Mass fraction of the gas 1 


\section{Conclusion}

In this work, we reviewed some results of thermodynamics of binary mixture. At equilibrium the energy of the two phases is given by an optimization problem on a convex set of constraints on extensive variables. The equilibrium energy is the inf-convolution of the energies of the two phases. In intensive parameters we proved that:

- for an immiscible mixture, the mixture energy is the convex hull of the energies;

- for a miscible mixture, the equilibrium energy is the inf-convolution of the phases energies.

Both operations transform respectively into max and plus operations in the Legendre space. We have thus put in evidence that the natural mathematical tool for studying mixture EOS is the theory of max-plus algebra.

The link with the Fast Legendre Transform algorithm has been investigated. The $O(N)$ algorithm that we provide ensures a good treatment of the boundaries. The whole development is applied to mixture EOS. In the case of a perfect gas mixture, the algorithm allows us to recover the profil of isothermal curves. In a like manner the Maxwell area rule method for a van der Waals fluid is recovered by computing the convex hull using the LT algorithm. Finally we propose the computation of a miscible mixture. The whole approach can also be applied to more complex EOS.

This work can be extended in many directions. To our opinion, the main application would be the correct treatment of tabulated EOS incorporated in Computational Fluid Dynamics (CFD) softwares. Usually, these tabulated EOS are approximated by polynomials. There is no mathematical reason that these polynomials preserve the convexity of the thermodynamical potentials. This is particularly critical in the mixture region, where the energy is convex but no more strictly convex. It can lead to loss of hyperbolicity and instabilites of the numerical model. Using the recently developed theory of max-plus algebra [Ma87, KM97] should lead to much more powerful and robust numerical techniques for approximating real EOS.

\section{REFERENCES}

[AFK07] Allaire, Grégoire and Faccanoni, Gloria and Kokh, Samuel. A strictly hyperbolic equilibrium phase transition model. C. R., Math., Acad. Sci. Paris. 344 (2007), no. 2, 135-140.

[Br89] Brenier, Yann. Un algorithme rapide pour le calcul de transformées de LegendreFenchel discrètes. C. R. Acad. Sci. Paris Sér. I Math. 308 (1989), no. 20, 587-589.

[Ca85] Callen, H. B.. Thermodynamics and an introduction to thermostatistics. Wiley and Sons, second edition (1985).

[Co96] Corrias, Lucilla. Fast Legendre-Fenchel transform and applications to HamiltonJacobi equations and conservation laws. SIAM J. Numer. Anal. 33 (1996), no. 4, 1534-1558.

[HS06] Helluy, Philippe and Seguin, Nicolas. Relaxation models of phase transition flows. M2AN Math. Model. Numer. Anal. 40 (2006), no. 2, 331-352.

[HL01] Hiriart-Urruty, Jean-Baptiste and Lemaréchal, Claude. Fundamentals of convex analysis. Grundlehren Text Editions. Springer-Verlag, Berlin, 2001.

[Ja01] Jaouen, S. Etude mathématique et numérique de stabilité pour des modèles hydrodynamiques avec transition de phase. PhD Thesis (2001). 
[KM97] Kolokoltsov, Vassili N. and Maslov, Victor P. Idempotent analysis and its applications. Mathematics and its Applications, 401. Kluwer Academic Publishers Group, Dordrecht, 1997. xii+305 pp.

[Lu96] Lucet, Yves. A fast computational algorithm for the Legendre-Fenchel transform. Comput. Optim. Appl.(1996), no. 1, 27-57.

[Lu98] Lucet, Yves. Faster than the fast Legendre transform, the linear-time Legendre transform. Numer. Algorithms 16 (1997), no. 2, 171-185.

[Ma87] Maslov, V. Méthodes opératorielles. (French) [Operator methods] Translated from the Russian by Djilali Embarek. Éditions Mir, Moscow, 1987. 708 pp.

[MaxPlus] INRIA Max-Plus project. Home page: http://maxplus.saclay.inria.fr/ index.fr.html

[MP89] Menikoff, Ralph and Plohr, Bradley J. The Riemann problem for fluid flow of real materials. Reviews of Modern Physics 61 (1989), no. 1, 75-130. 